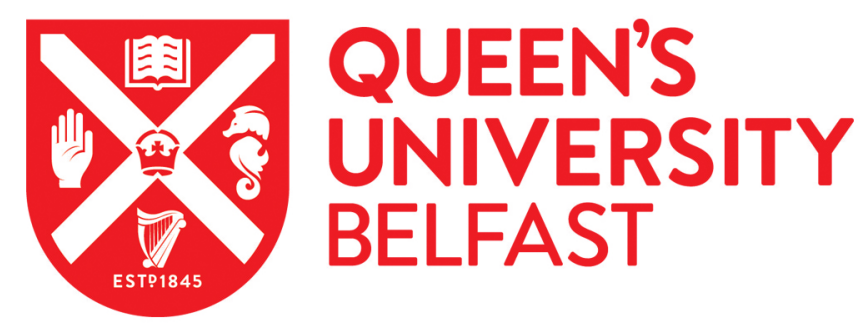

\title{
Early use of corticosteroids in infants with a clinical diagnosis of Pneumocystis jiroveci pneumonia in Malawi: a double-blind, randomised clinical trial
}

Newberry, L., O'Hare, B., Kennedy, N., Selman, A., Omar, S., Dawson, P., Stevenson, K., Nishihara, Y., Lissauer, S., \& Molyneux, E. (2017). Early use of corticosteroids in infants with a clinical diagnosis of Pneumocystis jiroveci pneumonia in Malawi: a double-blind, randomised clinical trial. Journal of Paediatrics and Child Health. https://doi.org/10.1080/20469047.2016.1260891

\section{Published in:}

Journal of Paediatrics and Child Health

\section{Document Version:}

Peer reviewed version

Queen's University Belfast - Research Portal:

Link to publication record in Queen's University Belfast Research Portal

\section{Publisher rights}

(c) 2016 John Wiley \& Sons. This work is made available online in accordance with the publisher's policies. Please refer to any applicable terms of use of the publisher.

\section{General rights}

Copyright for the publications made accessible via the Queen's University Belfast Research Portal is retained by the author(s) and / or other copyright owners and it is a condition of accessing these publications that users recognise and abide by the legal requirements associated with these rights.

Take down policy

The Research Portal is Queen's institutional repository that provides access to Queen's research output. Every effort has been made to ensure that content in the Research Portal does not infringe any person's rights, or applicable UK laws. If you discover content in the

Research Portal that you believe breaches copyright or violates any law, please contact openaccess@qub.ac.uk. 


\title{
Early steroid use in infants with a clinical diagnosis of Pneumocystis jiroveci pneumonia in Malawi- A double blinded randomized clinical trial
}

\author{
Abstract \\ Objectives \\ The aim of this study was to determine whether the early administration of adjuvant \\ corticosteroids in addition to standard treatment reduces mortality amongst infants with \\ vertically acquired HIV and clinically diagnosed PJP when co-infection with Cytomegalovirus \\ and other pathogens cannot be excluded.
}

Methods

We did a double-blinded placebo-controlled clinical trial of adjuvant prednisone treatment in HIV exposed infants aged 2 to 6 months admitted to Queen Elizabeth Central Hospital in Blantyre, Malawi clinically diagnosed as being infected with PJP. The protocol provided for the addition of prednisone to the treatment at 48 hours if there was clinical deterioration or an independent indication for steroid therapy. Oral trimethoprim-sulfamethoxazole therapy and full supportive treatment was given according to established guidelines. Primary outcomes for all patients included survival to hospital discharge and 6-month post-discharge survival.

\section{Results}

78 infants were enrolled between April 2012 to August 2014; 36 infants (46\%) were randomized to receive corticosteroids plus standard treatment with trimethoprim-sulfamethoxazole and 42 infants (54\%) received the standard treatment alone (placebo group). In an intention to treat analysis, the risk ratio of in-hospital mortality was 0.53 [95\% Confidence Interval (CI) 0.29-0.97, 
$\mathrm{p}=0.038$ ] in the corticosteroid group compared to the standard treatment group. The risk ratio of mortality at six months is 0.63 [95\% CI 0.41-0.95, $\mathrm{p}=0.029$ ]). Two children who received corticosteroids developed bloody stools while in hospital.

\section{Discussion}

The early use of corticosteroids in addition to conventional trimethoprim-sulfamethoxazole therapy in infants with a clinical diagnosis of PJP significantly reduced in hospital mortality and post discharge mortality at six months, compared with placebo.

Keywords: HIV, Pneumocystis jiroveci pneumonia, infant

\section{Introduction}

Pneumocystis jiroveci pneumonia (PJP) is a eukaryotic organism with features resembling a protozoa and fungi, phylogenetic analysis has demonstrated it to be a fungus. Exposure and colonisation occurs during the first few months of life and invasive disease may occur during this initial infection, especially in infants with severe T cell defects. ${ }^{1}$ It is the most common opportunistic infection in infants with vertically acquired HIV, it occurs in infants with low CD4 counts, ${ }^{2}$ and is the most common cause of death in HIV positive African infants less than six months of age, ${ }^{3}$ accounting for one third to half of all HIV related deaths in African infants. ${ }^{4}$ Clinical features include severe hypoxaemia, severe respiratory distress with a relatively clear chest or diffuse sign on auscultation and a low-grade fever. ${ }^{5}$ In the absence of diagnostic tests in most parts of Africa, many clinicians commence treatment, both trimethoprim-sulfamethoxazole (TMP/SMX), and often adjunctive corticosteroids, for PJP on the basis of clinical suspicion only. ${ }^{6}$ The rationale for steroids is that inflammation often worsens after treatment with high 
dose TMP/SMX is started and the patient may clinically deteriorate. A systematic review suggested a beneficial effect of adjunctive corticosteroids for adults with hypoxaemia, with a reduced risk of death and a reduced risk of requiring mechanical ventilation (RR 0.38). Only one study in infants was included and this showed the relative risk of death in hospital to be 0.81 but with wide confidence intervals. Therefore infants were not included in the authors’

recommendations for steroids as adjunctive treatment due to insufficient evidence. ${ }^{7}$ The included study randomized 100 infants with an average age of 3 months, exposed to HIV but not confirmed to be infected, diagnosed clinically with PJP to receive steroids, after 48 hours if there was clinical deterioration. They found patients in the prednisolone group had $43 \%$ better chance of survival but this was not statistically significant. ${ }^{8}$

There are a number of concerns about extrapolating the benefits in adults to infants in the absence of a randomised controlled trial. This includes concern about the immunosuppressive effects of administering steroids to infants who have an immature immune system as well as HIV related immunosuppression. HIV exposed infants with respiratory distress are often co-infected with viruses, particularly cytomegalovirus (CMV) whose role in the pathological process is unclear, but steroids may further immunosuppress this group of infants and contribute to the development of CMV pneumonia. CMV viraemia peaks around 3-4 months of age and is higher in HIV infected than HIV exposed infants. ${ }^{9}$ Williams and colleagues ${ }^{10}$ described 85 HIV and PJP infected infants in a pre ART era, of whom 48\% were co-infected with CMV. They found that PJP/CMV co-infected infants were more likely to require ventilation, and there was a trend towards poorer survival. They observed no benefit from adjunctive steroids on survival but raised the question if this was due to the high rate of coinfection with CMV. Thus there are concerns that steroids may negatively affect 
the clinical course if an infant is co-infected which may outweigh the potential benefit of steroids in PJP infected infants in a setting where CMV can neither be tested nor treated.

The aim of this study was to determine whether the early administration of adjuvant corticosteroids with high dose oral co-trimoxazole reduces mortality amongst infants with vertically acquired HIV and clinically diagnosed PJP when co-infection with CMV and other pathogens cannot be excluded.

\section{Methods}

\section{Study design}

This was a randomised double-blind placebo-controlled trial, conducted among HIV exposed infants aged 2 to 6 months admitted to hospital with severe respiratory distress and clinically diagnosed as being infected with PJP.

The study was carried out in the paediatric department of the Queen Elizabeth Central Hospital (QECH) in Blantyre, Malawi. The department has 300 beds and admits 25,000 children a year and there are about 80,000 emergency and outpatient attendances per annum. The QECH is an 1100 bedded tertiary, government hospital that serves the southern half of Malawi. It is the main teaching hospital of the medical school and is also the district hospital for the Blantyre region. All treatment is free. In 2011 Malawi introduced integrated antiretroviral/ prevention of mother to child transmission (ART/PMTCT guidelines) which integrated PMTCT services into maternal 
and child health services (MNCH). These guidelines included lifelong ART for all pregnant women regardless of WHO stage or CD4 count. ${ }^{11}$

\section{Study participants}

Infants aged two to six months of age with vertically acquired HIV or HIV exposure and clinical features of PJP were enrolled after full information in Chichewa or English and written consent from their guardians. PJP was defined as a disease that met all the following clinical criteria: vertically acquired HIV or HIV exposure, an oxygen requirement (saturations on air $<90 \%$ ) and severe respiratory distress and cough. Severe respiratory distress was defined as an infant with tachypnoea (respiratory rate $>60$ breaths/minute), tachycardia (heart rate $>140$ beats/min), subcostal and intercostal recessions and flaring of the alae nasi. Although not a requirement for a clinical diagnosis, history of low-grade fever, clear chest or absence of focal signs such as crepitations on auscultation were considered supportive of the clinical diagnosis of PJP.

Patients were excluded if they had a previously known allergy, hypersensitivity or other contraindication to corticosteroids or TMP/SMX. Infants were also excluded if they were previously treated for suspected PJP or if there was a delay starting steroids for greater than 24 hours after starting high dose TMP/SMX. Infants with clear clinical features of bronchiolitis and infants whose guardians did not give consent were excluded.

\section{Randomisation and masking}

Infants were randomised to one of two groups - standard care and the intervention group. Computer generated randomisation was completed in advance by an independent statistician 
prior to study activation. Infants were allocated to receive oral prednisone or placebo, TMP/SMX and supportive treatment. Allocation was concealed using numbered, sealed, opaque envelopes which were used in sequence and opened by the enrolling clinician after consent had been obtained. No stratification methods were employed. The placebo and study drug were identical when in white powder form and when reconstituted in water. The placebo was calcium carbonate. Parents, guardians, enrolling clinician, nurses reconstituting and administering the study drug, those assessing outcomes and those analysing the data were masked to the group assignment. The masking was assessed by an independent paediatrician.

\section{Procedures}

Treatment with TMP/SMX was commenced upon diagnosis of PJP pneumonia. Recruitment to the study (i.e. addition of either steroids or placebo) was done within 24 hours of diagnosis. The study medicine identified in the envelope was given according to the child's body weight. The steroid regime consisted of once daily oral prednisone at $2 \mathrm{mg} / \mathrm{kg}$ for 7 days, then $1 \mathrm{mg} / \mathrm{kg}$ for 7 days, then $0.5 \mathrm{mg} / \mathrm{kg}$ for 7 days for a total of 21 days.

The protocol provided for the addition of prednisone to the treatment at 48 hours if there was clinical deterioration or an independent indication for steroid therapy. The child would then stop the study solution and begin the prednisone course of $2 \mathrm{mg} / \mathrm{kg}$ for 7 days, then $1 \mathrm{mg} / \mathrm{kg}$ for 7 days, then $0.5 \mathrm{mg} / \mathrm{kg}$ for 7 days for a total of 21 days.

Children in both arms were commenced on high dose oral co-trimoxazole - Trimethoprim 8mg/kg/dose plus Sulphamethoxazole 60mg/kg/dose (TMP/SMX) twice daily for 21 days as per 
the WHO Guidelines. ${ }^{12}$ Given the suspected high rate of co-infection and to cover the main differential diagnosis of bacterial pneumonia, they were also commenced on parenteral benzylpenicillin (50,000 international units/kg/dose four times a day) and gentamicin (7.5mg/kg once a day) for at least 5 days. Additional oral zinc sulphate 10mg daily for seven days was given because of growing evidence that zinc sulphate improves case fatality among HIV exposed infants with pneumonia. ${ }^{13}$ Nasal prong oxygen was provided if oxygen saturations were less than 90\% in air. Oxygen was escalated according to our National Guidelines on the Care of the Infant and Neonate ${ }^{14}$ and infants who required bubble Continuous Positive Airway Pressure (bCPAP Pumani, $3^{\text {rd }}$ Stone Design, SanRafael, CA) received it depending on availability.

Upon recruitment, the following medical and clinical history was obtained using standardized forms by study staff: details about the present illness; demographic details; past medical history (including pre-existing conditions); birth history; HIV status; previous hospitalisations and visits to the local health centre. Temperature, pulse rate, respiratory rate (over one minute) and weight were recorded. The infant's arterial oxygen saturation was measured with an appropriately sized sensor and a Nellcor pulse oximeter (Welch Allyn Spot Vital Signs Devices, Skaneateles Falls, NY, USA) placed on a toe or finger, while the patient breathed room air. Measurements were recorded after stabilization for at least three minutes.

All HIV exposed infants had an ELISA and PCR for HIV done according to the National HIV guidelines for early infant diagnosis of HIV. ${ }^{11}$ Exposed infants who were clinically diagnosed as having PJP infection were started on anti-retroviral treatment during hospital admission, as they 
met the criteria for Presumed Severe HIV Disease (PSHD). The reason for starting without confirmation of HIV status was the delay receiving DNA PCR results of six to eight weeks.

All infants had a thick blood film tested for malaria parasites and packed cell volume estimated as standard care. Infants had 1-2 mls of venous blood taken for blood culture prior to commencing antibiotics. The patients were re-evaluated twice daily until discharge from high dependency and daily until discharge from hospital.

\section{Follow up post discharge}

After hospital discharge infants were followed up by the study clinicians at one, three and six months to assess survival and monitor adverse events.

\section{Outcomes}

The primary outcomes for all patients were survival to hospital discharge and 6-month posthospital discharge survival. Adverse events related to the antibiotics or corticosteroids were documented and graded as mild, moderate and severe.

\section{Sample Size and Statistical Analysis}

Hospital mortality in sub-Saharan African region for HIV positive PJP infected infants ranges from 20-63\%. ${ }^{15}$ The most recent published estimate of mortality from PJP in HIV positive Malawian infants is $63 \% .{ }^{5}$ This study was done prior to the introduction of bCPAP and before antiretroviral treatment was widely available and included a small number of infants (16) with confirmed PJP pneumonia. A study from a tertiary care centre in South Africa described a 
mortality rate of $27 \% .{ }^{16}$ We chose a conservative figure of $45 \%$ for the purposes of sample size calculation. We estimated that corticosteroids could reduce mortality by $20 \%$ as the study by Terblanche and colleagues ${ }^{8}$ showed a relative risk of mortality of 0.81 in those treated with steroids compared to those not given steroids. With a two-sided alpha level of 0.05, a total enrolment of 200 patients was needed to attain a power of $80 \%$ to detect an absolute reduction of $20 \%$ in mortality in the corticosteroid group.

Data were expressed as means and standard deviations. We used the Mann-Whitney test for continuous variables and the chi-square test for categorical variables. A two-sided P value of less than 0.05 was considered to indicate statistical significance. Intention to treat analysis (ITTA) was used as well as a treatment-only per protocol analysis (PPA). Within PPA, patients in both the placebo and treatment group who received prednisone after randomisation were disregarded in the analysis. We used the Kaplan-Meier method to estimate time-to-event distributions, which were compared with the use of the log-rank test as well as a cox-proportional hazard ratio. Analyses were performed with STATA, version 12.0.

Ethical approval was obtained from the College of Medicine Research and Ethics Committee. ${ }^{17}$ The study was registered at the US National Institutes of Health (ClinicalTrials.gov) \#NCT02149433. An independent safety-data monitoring committee reviewed data on safety and efficacy at predetermined intervals.

\section{Results}


Infants were enrolled from April 5, 2012 to August 28, 2014. The study was unable to recruit the sample size over this time as originally planned, perhaps due to the falling numbers of new HIV infections in infants being born, and to the routine use of TMP/SMX preventive therapy for HIVexposed infants which is very effective in preventing PJP. After 2.5 years of study, only 78 eligible infants had been enrolled following informed consent. Due to difficulties in continuation of the study, recruitment of even 100 participants would take at least one more year, it was decided to provide interim analysis of outcome data to the DSMB. Given the slower than expected recruitment to the study and that the interim analysis reported a difference in mortality of more than $30 \%$ between the two groups, a difference that was statistically significant, it was agreed to stop the study

Study enrolment, randomization and follow-up are shown in the trial profile in Figure 1. 116 were screened for eligibility: 8 refused consent and 30 were ineligible for enrollment. 78 eligible infants were enrolled and randomly assigned to each of the two study groups. 36 infants (46\%) were randomized to receive corticosteroids, TMP/SMX and supportive treatment and 42 infants (54\%) received the TMP/SMX and supportive treatment alone (placebo group). Two infants from the standard treatment group and three infants from the corticosteroid group were given steroids due to clinical deterioration per protocol provision. The two infants from the standard group who receive corticosteroids both died while in hospital. Of the three children from the corticosteroid group who were taken off the study solution and given known corticosteroids, two infants survived to hospital discharge and one died. Of the two infants who survived; one was lost to follow-up and the other survived to six months 
All infants were followed to discharge from the hospital or in-hospital demise. After discharge from the hospital, five infants (6\%) were lost to follow-up at six months: three infants from the corticosteroid arm and two from the standard treatment arm.

\section{Demographic and Clinical Characteristics}

The base-line characteristics of the two treatment groups were similar (Table 1). The mean age was $3.5 \pm 1.1$ months, and $42 \%$ were male. Clinical signs and symptoms were consistent with previous findings from published series of patients with a clinical diagnosis of PJP pneumonia including hypoxia, tachypnea and clear lungs. 27 mothers of recruited infants (34.6\%) knew that they were HIV positive during pregnancy. 16 mothers (20.5\%) gave their infants daily nevirapine prophylaxis during the first six weeks of life and 9 infants (11.5\%) received daily prophylactic TMP 4mg/kg per day plus SMX 30mg/kg per day after six weeks of life according to the National HIV Guidelines. ${ }^{[13]}$ None of the recruited infants presented to hospital with a DNA-PCR result and none were on anti-retroviral medication at the time of admission.

All recruited infants were HIV exposed. 47 infants (60.2\%) were tested for HIV with a DNAPCR test during admission. The other infants were not tested for HIV with DNA-PCR because they died before the tests could be done. 37 infants (47.4\%) had a positive HIV DNA PCR test and 10 infants (12.8\%) had a negative HIV DNA PCR test. Forty-one infants (52.6\%) survived and qualified to start antiretroviral treatment. 
bCPAP was started on 31 infants (40.8\%) of all recruited infants. The most common reason for not starting a child on bCPAP was lack of available equipment. There was no statistical difference between the groups in the number managed on bCPAP.

\section{Outcomes}

The main outcome results for both the intention to treat analysis and per protocol analysis are reported in Table 2. In an intention to treat analysis, 10 of 36 infants (27.8\%) who received corticosteroids died compared to 22 of 42 infants (52.4\%) who received standard treatment alone. The risk ratio of in-hospital mortality was 0.53 [95\% Confidence Interval (CI) 0.29-0.97, $\mathrm{p}$ value $=0.038$ ] in the corticosteroid group compared to the standard treatment group. At six months post-hospital discharge, 15 of the 33 infants (45.5\%) who received steroids died compared to 29 out of 40 infants who received the standard treatment alone (Risk ratio 0.63 (95\% CI 0.41-0.95, p value $=0.029)$.

In the per protocol analysis, 9 of 36 infants (27.3\%) who received corticosteroids died compared to 20 of 40 infants (50.0\%) who received standard treatment alone. The risk ratio of in-hospital mortality was 0.55 [95\% Confidence Interval (CI) 0.29-1.03, p value $=0.062$ ] in the corticosteroid group compared to the standard treatment group. At six months post-hospital discharge, 13 of 30 infants (45.5\%) who received steroids died compared to 27 out of 38 infants (71.1\%) who received the standard treatment alone (Risk ratio of 0.61 (95\% CI 0.39-0.96, p value=0.034)).

Figure 2 demonstrates that patients in the prednisone group had a 53\% better chance of survival at six months post-enrolment: Cox proportional hazard ratio 0.47 (95\% CI $0.24-0.92, \mathrm{p}=0.028)$ 
according to the intention to treat analysis.

\section{Adverse events}

Two children who received corticosteroids developed bloody stools while in hospital. The bloody stools resolved within 2-3 days in one child who survived to six-month post-discharge. The other child died while in hospital from respiratory compromise.

\section{Discussion}

In this double-blind, randomized trial, the early use of corticosteroids in addition to conventional TMP/SMX and supportive treatment in infants with a clinical diagnosis of PJP significantly reduced mortality, as compared with placebo. In a resource poor setting, where accurate diagnostics may not be available, our findings demonstrate that it is safe and beneficial to give corticosteroids in children who have clinical findings suspicious for PJP.

The Revised WHO classification and treatment of pneumonia in children at health facilities in resource poor settings ${ }^{12}$ now recommends empiric TMP/SMX treatment for suspected PJP as an additional treatment for HIV-infected and -exposed infants aged from 2 months up to 1 year with chest in-drawings or severe pneumonia. However, the guidelines do not mention whether or not adjunctive corticosteroids should be used as well. Our findings suggest that empiric treatment with corticosteroids should be included in these guidelines for those HIV-infected and exposed infants who have clinical findings suspicious for PJP infection. 
Infants infected with PJP may also be infected with CMV. CMV is usually acquired during the first year of life in African children and HIV infected infants are at increased risk of developing CMV pneumonia. The gold standard in terms of diagnosis is evidence of pathology on lung biopsy as identifying CMV in respiratory specimens may or may not be associated with pneumonia. ${ }^{9}$ In a systematic review of nine paediatric autopsy studies, PJP was identified in 23\% and CMV in $22 \%$ of autopsies on HIV positive infants, with high rates of coinfection. ${ }^{18}$ Goussard and colleagues ${ }^{19}$ examined lung tissue either by biopsy or using post mortem tissue of 25 HIV infected infants admitted to an intensive care unit with severe hypoxia and found CMV to be present in 36\% of patients, another 36\% had dual infection and 24\% had PJP alone.

It is therefore highly likely that infants in both arms of our study had CMV infection and pneumonia but in the absence of the ability to diagnose or treat CMV it appears that the addition of adjunctive steroids in HIV positive and exposed infants with a clinical diagnosis of PJP nonetheless reduced the mortality at discharge and at six months.

Morrow and colleagues ${ }^{15}$ found that $74 \%$ of HIV and PJP (diagnosed molecularly) infected infants had CMV viraemia. A study comparing levels of viraemia between HIV exposed and infected infants with and without pneumonia found that a level greater than 4.1 log copies per ml identified infants with likely CMV pneumonia. ${ }^{9}$ Kitchin and colleagues ${ }^{20}$ also used the cut off of 4 log copies per ml to determine which infants they would discontinue the empiric gancyclovir treatment which they commenced on all HIV exposed infants who presented with acute respiratory failure. Appropriate diagnosis and management of CMV in these patients would be optimal. 
More important is ensuring that all women who are pregnant know their status and all infants are commenced on prophylactic trimethoprim -sulfamethoxazole. In this cohort, only 35\% of the mothers were aware of their HIV diagnosis and of those who were aware, only $33 \%$ of their infants received prophylactic trimethoprim-sulfamethoxazole. This is a biased sample as these infants presented with symptoms in keeping with PJP pneumonia. At a population level, in 2014, 79\% of pregnant women in Malawi knew their HIV status, 93\% of babies were commenced on nevirapine prophylaxis and $85 \%$ of babies were started on prophylactic TMP/SMX. ${ }^{21}$

Limitations of our study include not having a complete set of microbiologically confirmed diagnosis of PJP or CMV. Strengths of the study include that it was done in a pragmatic way which reflects the situation where the vast majority of infants who are infected with HIV, PJP and CMV are managed. Although the study was stopped early, we believe the results to be robust and continuing enrolment to complete the sample size would not have affected the outcome of the trial.

\section{Conclusions and recommendations}

Infants with a clinical diagnosis of PJP should be treated with adjunctive steroids even in the absence of information about coinfection with CMV. Current WHO guidelines should consider advising adjunctive steroids in addition to empiric TMP/SMX treatment for suspected PJP in infants in a resource poor setting. Every effort should be made to ensure all women know their 
HIV status in pregnancy and at delivery and to ensure all exposed babies receive prophylaxis to prevent PJP infection.

\section{Author contributions}

EM, NK, BO, SL conceived the study. LN, EM, NK, BO, AS and SL designed the study. LN, AS, SO, PD, KS, YN were responsible for data collection. LN, EM, NK, BO and YN undertook data analysis. BO and EM provided the literature search. LN wrote the first draft of the paper. All authors contributed to the interpretation of the data, writing of the report, and approved the final manuscript.

\section{Declaration of interests}

We declare no competing interests.

\section{Financial support}

There was no funding source for this study.

\section{Data Safety and Monitoring Board}

Steve Graham, MBBS and Aisleen Bennett, MBBS. The Data and Safety Monitoring Board reviewed two formal interim analyses and regular reports of our primary composite outcome as well as serious adverse events.

\section{References}

1 Pyrgos V, Shoham S, Roilides E, Walsh, TJ. Pneumocystis pneumonia in children. Paediatr Respir Rev. 2009; 10(4):192-198. 
Kovacs A, Frederick T, Church J, Eller A, Oxtoby M, Mascola L. CD4 T-lymphocyte counts and Pneumocystis carinii pneumonia in pediatric HIV infection. JAMA. 1991; 265(13):1698-703.

Zar HJ. Pneumonia in HIV-infected and HIV-uninfected children in developing countries: Epidemiology, clinical features, and management. Curr Opin Pulm Med. 2004; 10:176-182. Graham SM. Non-tuberculosis opportunistic infections and other lung diseases in HIV-infected infants and children. Int J Tuberc Lung Dis. 2005; 9(6):592-602. Graham SM, Mtitimila EI, Kamanga HS, Walsh AL, Hart CA, Molyneux ME. Clinical presentation and outcome of Pneumocystis carinii penumonia in Malawian children. Lancet. 2000; 355:369-73.

6 Zar HJ, Jeena P, Argent A, Gie R, Madhi SA; Working Groups of the Paediatric Assembly of the South African Thoracic Society. Diagnosis and management of community-acquired pneumonia in childhood--South African Thoracic Society Guidelines. S Afr Med J. 2005; 95:977-81, 984-90.

7 Ewald H, Raatz H, Boscacci R, Furrer H, Bucher HC, Briel M. Adjunctive corticosteroids for Pneumocystis jiroveci pneumonia in patients with HIV-infection. Cochrane Database Syst Rev. 2015;4:(CD006150.) Terblanche AJ, Green RJ, Rheeder P, Wittenberg DF. Adjunctive corticosteroid treatment of clinical Pneumocystis jiroveci pneumonia in infants less than 18 months of age--a randomised controlled trial. S Afr Med J. 2008. 98(4):287-90. Hsiao NY, Zampoli M, Morrow B, Zar HJ, Hardie D. Cytomegalovirus viraemia in HIV exposed and infected infants: prevalence and clinical utility for diagnosing CMV pneumonia. J Clin Virol. 2013. 58(1)74-80. Williams AJ, Duong T, McNally LM, et al. Pneumocystis carinii pneumonia and cytomegalovirus infection in children with vertically acquired HIV infection. AIDS. 2001. 15(3):335-9. MoH [Malawi]. Clinical Management of HIV in Children and Adults. Malawi Ministry of Health, Lilongwe, Malawi; 2011. WHO. Revised WHO classification and treatment of pneumonia in children at health facilities: evidence summaries. World Health Organization, Geneva; 2014. Srinivasan MG, Ndeezi G, Mboijana CK, et al. Zinc adjunct therapy reduces case fatality in severe childhood pneumonia: a randomized double blind placebocontrolled trial. in BMC Medicine. 2012; 10:14.

14 MoH-RHU [Malawi]. Care of the Infant and Neonate (COIN). Malawi Ministry of Health, Reproductive Health Unit, Lilongwe, Malawi; 2015 Morrow BM, Samuel CM, Zampoli M, Whitelaw A, Zar HJ. Pneumocystis pneumonia in South African children diagnosed by molecular methods. BMC Res Notes. 2014; 7:26.

16 Ruffini DD, Madhi SA. The high burden of Pneumocystis carinii pneumonia in African HIV-1-infected children hospitalized for severe pneumonia. AIDS. 2002. 16(1):105-12. COMREC, College of Medicine Research Ethics Committee. Available from: http://www.medcol.mw/comrec/ [Accessed 19th June 2015]. in Africa. Curr Opin Pulm Med. 2013. 19(3):229-237. 
Goussard P, Kling S, Gie RP, et al. CMV pneumonia in HIV-infected ventilated infants. Pediatr Pulmonol. 2010. 45(7):650-655.

20 Kitchin OP, Masekela R, Becker P, Moodley T, Risenga SM, Green RJ. Outcome of human immunodeficiency virus-exposed and -infected children admitted to a pediatric intensive care unit for respiratory failure. Pediatr Crit Care Med. 2012. 13(5):516-9.

21 Government of Malawi. Malawi AIDS Response Progress Report. Malawi Ministry of Health, Lilongwe, Malawi; 2015.

22 Punpanich W, Groome M, Muhe L, Qazi SA, Madhi SA. Antibiotic and systemic therapies for pneumonia in human immunodeficiency virus (HIV)-infected and HIVexposed children. J Infect Dev Ctries. 2012. 6(2):109-119.

23 Bye M, Cairns-Bazarian AM, Ewig JM. Markedly reduced mortality associated with corticosteroid therapy of Pneumocystis carinii pneumonia in children with acquired immunodeficiency syndrome. Arch Pediatr Adolesc Med. 1994. 148(6):638-641.

24 Sleasman JW, Hemenway C, Klein AS, Barrett DJ. Corticosteroids improve survival of children with AIDS and Pneumocystis carinii pneumonia. Am J Dis Child. 1993. 147(1): 30-4. 


\section{Table 1 Baseline Characteristics}

\begin{tabular}{|c|c|c|}
\hline & $\begin{array}{r}\text { Corticosteroid Group } \\
\mathrm{N}=36(\%)\end{array}$ & $\begin{array}{r}\text { Placebo Group } \\
\mathrm{N}=42(\%)\end{array}$ \\
\hline Male & $12(33)$ & $21(50)$ \\
\hline Age (median, month) \pm SD & $3.1(2.7-3.9)$ & $3.4(2.9-4.4)$ \\
\hline Weight (median, g) \pm SD & $5100(4400-5700)$ & $4650(3800-5300)$ \\
\hline $\begin{array}{l}\text { Mother knew she was HIV positive } \\
\text { during pregnancy }\end{array}$ & 11(31) & 16(38) \\
\hline $\begin{array}{l}\text { Infant was given Neviraprine } \\
\text { prophylaxis }\end{array}$ & $5(14)$ & $11(26)$ \\
\hline $\begin{array}{l}\text { Infant was started on trimethoprim- } \\
\text { sulfamethoxazole prophylaxis }\end{array}$ & $3(8)$ & $6(14)$ \\
\hline \multicolumn{3}{|l|}{$\begin{array}{l}\text { Infant HIV Status Determined after } \\
\text { Admission }\end{array}$} \\
\hline HIV exposed, DNA-PCR Reactive & $21(58)$ & $16(38)$ \\
\hline HIV exposed, DNA-PCR Negative & $4(11)$ & $6(14)$ \\
\hline HIV exposed, DNA-PCR not done & $11(31)$ & $20(48)$ \\
\hline \multicolumn{3}{|l|}{$\begin{array}{l}\text { Clinical Characteristics at } \\
\text { Enrollment }\end{array}$} \\
\hline Respiratory Rate (median) \pm SD & $66(60-78)$ & $70(62-80)$ \\
\hline $\begin{array}{l}\text { Oxygen saturation on room air } \\
\text { (median, \%) } \pm \text { SD }\end{array}$ & $68(60-78)$ & $70(62-80)$ \\
\hline $\begin{array}{l}\text { Temperature (median, }{ }^{\circ} \text { Celsius) } \pm \text { SD } \\
\text { Chest Exam Findings }\end{array}$ & 37.7(36.8-38.3) & $37.9(37.6-38.3)$ \\
\hline Clear lungs & 24(67) & $30(71)$ \\
\hline Bilateral crepitations & $12(33)$ & 12(29) \\
\hline Packed Cell Volume (median, \%) \pm SD & $34(32-39)$ & $34(29-36)$ \\
\hline Placed on Continuous Positive Airway & & \\
\hline Pressure (CPAP) & $16 / 35(46)$ & 15/41(37) \\
\hline
\end{tabular}


Table 2- Primary Study Outcomes

\begin{tabular}{|c|c|c|c|c|c|}
\hline & Prednisone Group & Placebo Group & $\begin{array}{l}\text { Risk } \\
\text { Ratio }\end{array}$ & $\begin{array}{l}95 \% \\
\text { Confidence } \\
\text { Interval }\end{array}$ & $P$ Value \\
\hline \multicolumn{6}{|c|}{ Intention to Treat Analysis } \\
\hline $\begin{array}{l}\text { In-hospital } \\
\text { Mortality }\end{array}$ & $10 / 36(28 \%)$ & $22 / 42(52 \%)$ & 0.53 & $(0.29-0.97)$ & 0.038 \\
\hline $\begin{array}{l}\text { Six Months } \\
\text { Post-Discharge } \\
\text { Mortality }\end{array}$ & 15/33 (46\%) & $29 / 40(73 \%)$ & 0.63 & $(0.41-0.95)$ & 0.029 \\
\hline \multicolumn{6}{|c|}{ Per Protocol Analysis } \\
\hline $\begin{array}{l}\text { In-hospital } \\
\text { Mortality }\end{array}$ & 9/33 (27\%) & $20 / 40(50 \%)$ & 0.55 & $(0.29-1.03)$ & 0.062 \\
\hline $\begin{array}{l}\text { Six Months } \\
\text { Post-Discharge } \\
\text { Mortality }\end{array}$ & $13 / 30(46 \%)$ & 27/38 (71\%) & 0.61 & $(0.39-0.96)$ & 0.034 \\
\hline
\end{tabular}

Figure 1- Trial Profile

Figure 2- Kaplan Meier Plot 